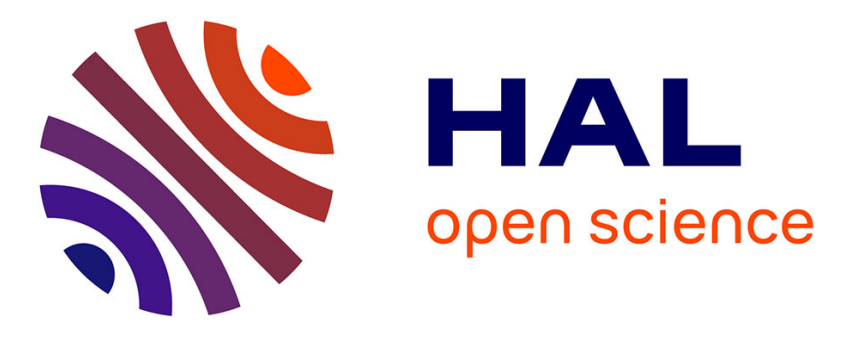

\title{
Three SCC-based Emptiness Checks for Generalized Büchi Automata
}

\author{
Etienne Renault, Alexandre Duret-Lutz, Fabrice Kordon, Denis Poitrenaud
}

\section{To cite this version:}

Etienne Renault, Alexandre Duret-Lutz, Fabrice Kordon, Denis Poitrenaud. Three SCC-based Emptiness Checks for Generalized Büchi Automata. 19th International Conference on Logic for Programming, Artificial Intelligence, and Reasoning (LPAR'13), Dec 2013, Stellenbosch, South Africa. pp.668682, 10.1007/978-3-642-45221-5_44. hal-01066992

\section{HAL Id: hal-01066992 https://hal.science/hal-01066992}

Submitted on 22 Sep 2014

HAL is a multi-disciplinary open access archive for the deposit and dissemination of scientific research documents, whether they are published or not. The documents may come from teaching and research institutions in France or abroad, or from public or private research centers.
L'archive ouverte pluridisciplinaire HAL, est destinée au dépôt et à la diffusion de documents scientifiques de niveau recherche, publiés ou non, émanant des établissements d'enseignement et de recherche français ou étrangers, des laboratoires publics ou privés. 


\title{
Three SCC-based Emptiness Checks for Generalized Büchi Automata
}

\author{
E. Renault ${ }^{1,2}$, A. Duret-Lutz ${ }^{1}$, F. Kordon ${ }^{2}$, D. Poitrenaud ${ }^{2,3}$ \\ 1 LRDE, EPITA, Kremlin-Bicêtre, France. \\ ${ }^{2}$ LIP6/MoVe, Université Pierre \& Marie Curie, Paris, France. \\ 3 Université Paris Descartes, Paris, France
}

\begin{abstract}
The automata-theoretic approach for the verification of linear time properties involves checking the emptiness of a Büchi automaton. However generalized Büchi automata, with multiple acceptance sets, are preferred when verifying under weak fairness hypotheses. Existing emptiness checks for which the complexity is independent of the number of acceptance sets are all based on the enumeration of Strongly Connected Components (SCCs).

In this paper, we review the state of the art SCC enumeration algorithms to study how they can be turned into emptiness checks. This leads us to define two new emptiness check algorithms (one of them based on the Union-Find data structure), introduce new optimizations, and show that one of these can be of benefit to a classic SCCs enumeration algorithm. We have implemented all these variants to compare their relative performances and the overhead induced by the emptiness check compared to the corresponding SCCs enumeration algorithm. Our experiments shows that these three algorithms are comparable.
\end{abstract}

\section{Introduction}

The automata-theoretic approach to explicit LTL model checking explores the product between two $\omega$-automata: one automaton that represents the system, and the other that represents (the negation of) the property to check on this system. This product corresponds to the intersection between the executions of the system and the behaviors disallowed by the property. The property is verified by the system if this product is empty.

Usually, a Büchi automaton is used to represent the property, and a Kripke structure represents the model. However, it is possible to use generalized Büchi automata (with several acceptance sets) to represent the property in a more concise way, and such generalized acceptance condition can also be used on the model to express weak fairness hypotheses on the system. In this work, we further generalize the above approach using Transition-based Generalized Büchi Automata (TGBA).

An emptiness check is an algorithm deciding whether such an automaton is empty. A Büchi automaton is non-empty if it accepts an infinite word, i.e., if it contains a lasso-shaped run: a finite prefix followed by an accepting cycle. Most 
explicit emptiness checks are based on a DFS exploration of the automaton; they can be classified in two families. Nested Depth First Search algorithms [3] use a second DFS to detect the accepting cycle: if the automaton has multiple acceptance sets, this approach requires either a degeneralization, or multiple nested DFS. The second family are algorithms based on the enumeration of Strongly Connected Components (SCC), to find SCCs that contain accepting cycles. In these algorithms the number of times a state or transitions is visited is independent on the number of acceptance sets.

In this paper, we review the existing SCC enumeration algorithms to study how they can be adapted to become emptiness checks. To be of practical use in a model checker, we would like such emptiness checks to:

- support generalized Büchi acceptance [5, 12] (without requiring a degeneralization, or multiple passes on the automaton),

- support an on-the-fly construction of the automaton so that we do not need to construct unexplored parts of the product,

- be compatible with the bit-state hashing [15] and state-space caching [13] techniques to deal cases where memory is a critical resource.

We focus on three SCC algorithms which we shall refer to as Tarjan [19], Dijkstra [6], Gabow [8]. Tarjan is the most well-known algorithm to compute SCC and it has been extended by Geldenhuys and Valmari [11] to check the emptiness of (non-generalized) Büchi automata. Dijkstra's SCC-enumeration algorithm is a little less known, but has served as the base for several generalized emptiness checks $[12,5,1,10]$. Essentially, both these algorithms partition the set of states according to the SCCs, and have a complexity that is linear with respect to the size of the graph. An efficient data structure to deal with the construction of a partition is the Union-Find [20] and Gabow [8] has suggested an algorithm to label the SCCs of a graph using such a data structure; in this context the number of Union-Find operations is linear in the size of the graph, and the amortized time-complexity of these operations is quasi-constant (related to the inverse of the Ackermann function) in the worst case. To our knowledge, this suggested algorithm, which we call Gabow ${ }^{4}$, has never been experimented to compute SCCs, let alone to perform an emptiness check.

Our contributions are as follows. (1) We show how to adapt Tarjan's algorithm to perform a generalized emptiness check. (2) We suggest an optimization of Dijkstra's algorithm that also benefits all the emptiness checks based on this algorithm. (3) We extend Gabow's idea to implement a Union-Find-based emptiness check. (4) Moreover we show how to adjust all these algorithms to support bit-state hashing and state-space caching.

While our experiments shows that there is no algorithm that clearly outperforms the others, we believe that having the choice between these three differents schemes might prove useful to devise new extensions (such as parallel model checking).

\footnotetext{
${ }^{4}$ Beware! The main algorithm of Gabow's paper [8] is a reinvention of Dijkstra's algorithm. Cf. http://www.cs.colorado.edu/ hal/Papers/DFS/pbDFShistory.html. What we call Gabow's algorithm here is the idea evoked on page 109 of that paper.
} 
This paper is organized as follows. Section 2 defines TGBAs and introduces our notations. Sections 3-5 successively present Tarjan's, Dijkstra's, and Gabow's algorithms and discuss how that can be extended to perform emptiness checks. Section 6 discusses the compatibility of these algorithms with the bit-state hashing and state-space caching techniques. Finally Section 7 provides experimental data to compare all these algorithms.

\section{Preliminaries}

Let $G=\left\langle Q, q^{0}, \delta\right\rangle$ be a directed graph with $Q$ the set of states, $q^{0}$ the initial state, and $\delta \subseteq Q \times Q$ the set of transitions.

A path of length $n \geq 1$ between two states $q, q^{\prime} \in Q$ is a finite sequence of edges $\rho=\left(s_{1}, s_{2}\right)\left(s_{2}, s_{3}\right) \ldots\left(s_{n}, s_{n+1}\right)$ with $s_{1}=q$ and $s_{n+1}=q^{\prime}$. We denote the existence of such a path by $q \rightsquigarrow q^{\prime}$. When $q=q^{\prime}$ the path is a cycle.

A non-empty set $S \subseteq Q$ is a Strongly Connected Component (SCC) iff $\forall s, s^{\prime} \in S, s \neq s^{\prime} \Rightarrow s \rightsquigarrow s^{\prime}$ and $S$ is maximal w.r.t. inclusion. A trivial $S C C$ is a state without self-loop.

A $T G B A$ is a tuple $A=\left\langle Q, q^{0}, \delta, \mathcal{F}, f\right\rangle$ where $\mathcal{F}$ is a finite set of acceptance marks and $f: \delta \mapsto 2^{\mathcal{F}}$ labels each transition of the directed graph $\left\langle Q, q^{0}, \delta\right\rangle$ by a set of acceptance marks. Let us note that in a real model checker, transitions (or states) of the automata would be labeled by atomic propositions, but we omit this information as it is not pertinent to emptiness check algorithms.

A degeneralization process can transform any TGBA with $n$ states and $m$ acceptances marks into an equivalent TGBA with one acceptance mark and at most $n m$ states.

An SCC $S \subseteq Q$ is accepting iff $\bigcup_{t \in(S \times S) \cap \delta}\{f(t)\}=\mathcal{F}$. A TGBA is non-empty iff there is a path from $q^{0}$ to an accepting SCC.

All the algorithms we consider are based on a DFS of a TGBA and we can present them by specializing the generic DFS algorithm of Algo. 1. This algorithm is slightly more complex than the average DFS, as we will use it in various settings. The $d f s$ variable is the stack of the DFS algorithm and stores: a set $a c c$ of acceptance marks labeling the transition leading to the state pos, and set succ of the unexplored successors of this state. The state pos is actually represented by a Position, which shall be defined differently in each algorithm.

Each state is either LIVE, DEAD, or UNKNOWN. A state is UNKNOWN until it has been explored by the DFS, then it becomes LIVE. A state may only become DEAD after all the successors of the SCC it belongs to have been visited. Maintaining this status will be done by each algorithm by implementing the following methods:

- GET_STATUS: returns the status of a state;

- PUSH: called for any newly visited state, it should mark that state as LIVE;

- UPDATE: called every time a back-edge (i.e., a transition leading to a LIVE state) is found, this function detects a transition closing a cycle;

- POP: called every time the DFS backtracks a state. When the last state of an SCC is being popped, all the states in its SCC must be marked as DEAD 


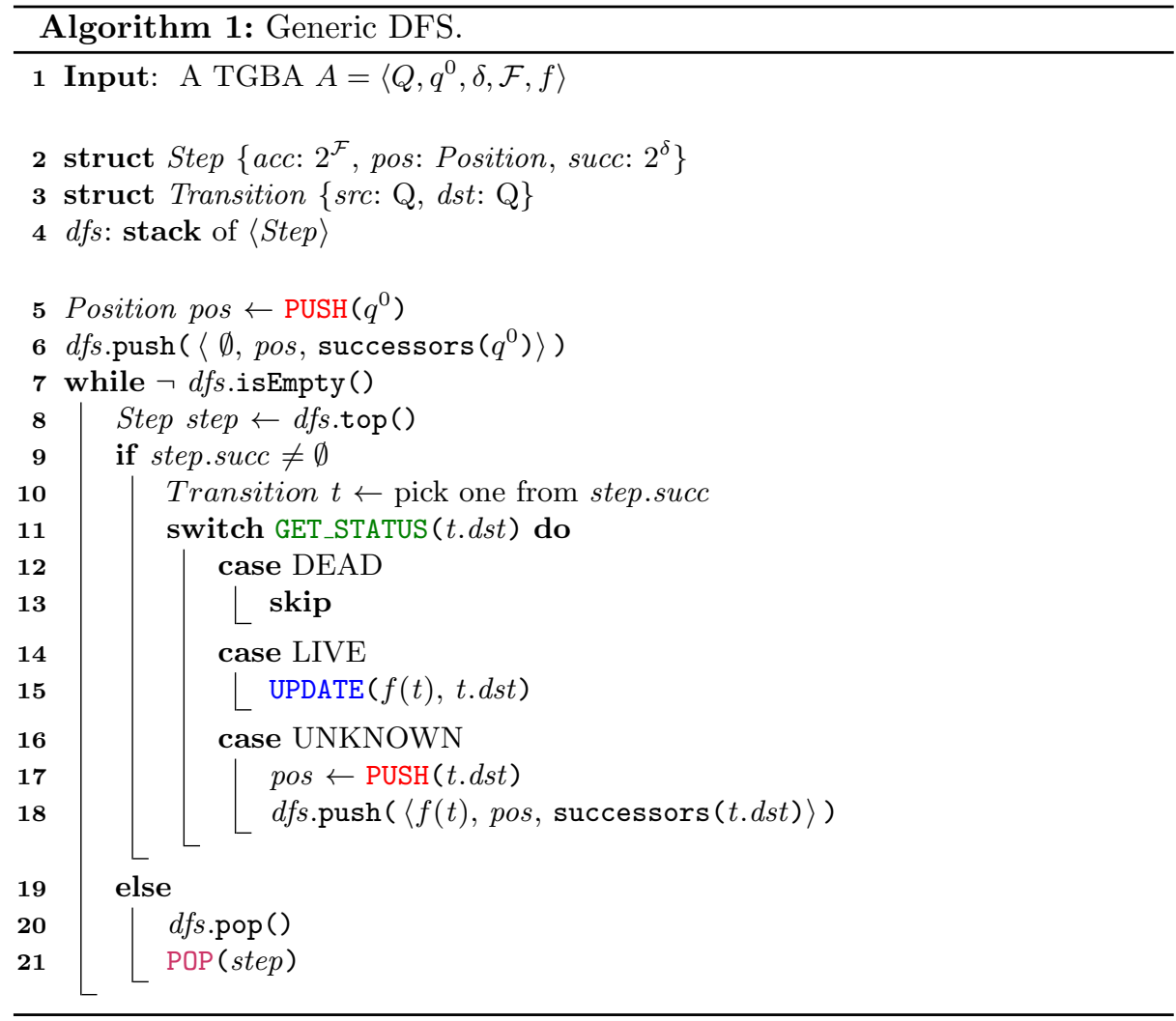

by POP. We call such a last state the root of the SCC (notice that this root may depend on the order in which the transitions are visited).

\section{Tarjan-based Algorithm}

\subsection{SCC Computation}

In Tarjan's original algorithm [19], each state is associated to two numbers: a DFS number (indicating the order in which the states has been visited by the DFS), and a lowlink. Initially, this lowlink is equal to the DFS number, but each time a transition is backtracked (i.e., during UPDATE or POP) the lowlink of the source is updated to the DFS number (for UPDATE) or to the lowlink (for POP) of the destination if it is smaller. An SCC root is detected during POP as a state whose lowlink is equal to the DFS number.

A usual optimization of POP is based on the fact that when a root is popped, the (outside) states that are successors of this SCC have already been marked as DEAD. Consequently, if the set of LIVE states is stored as a stack, then all the states of the current SCC are on this stack between the position of the root 


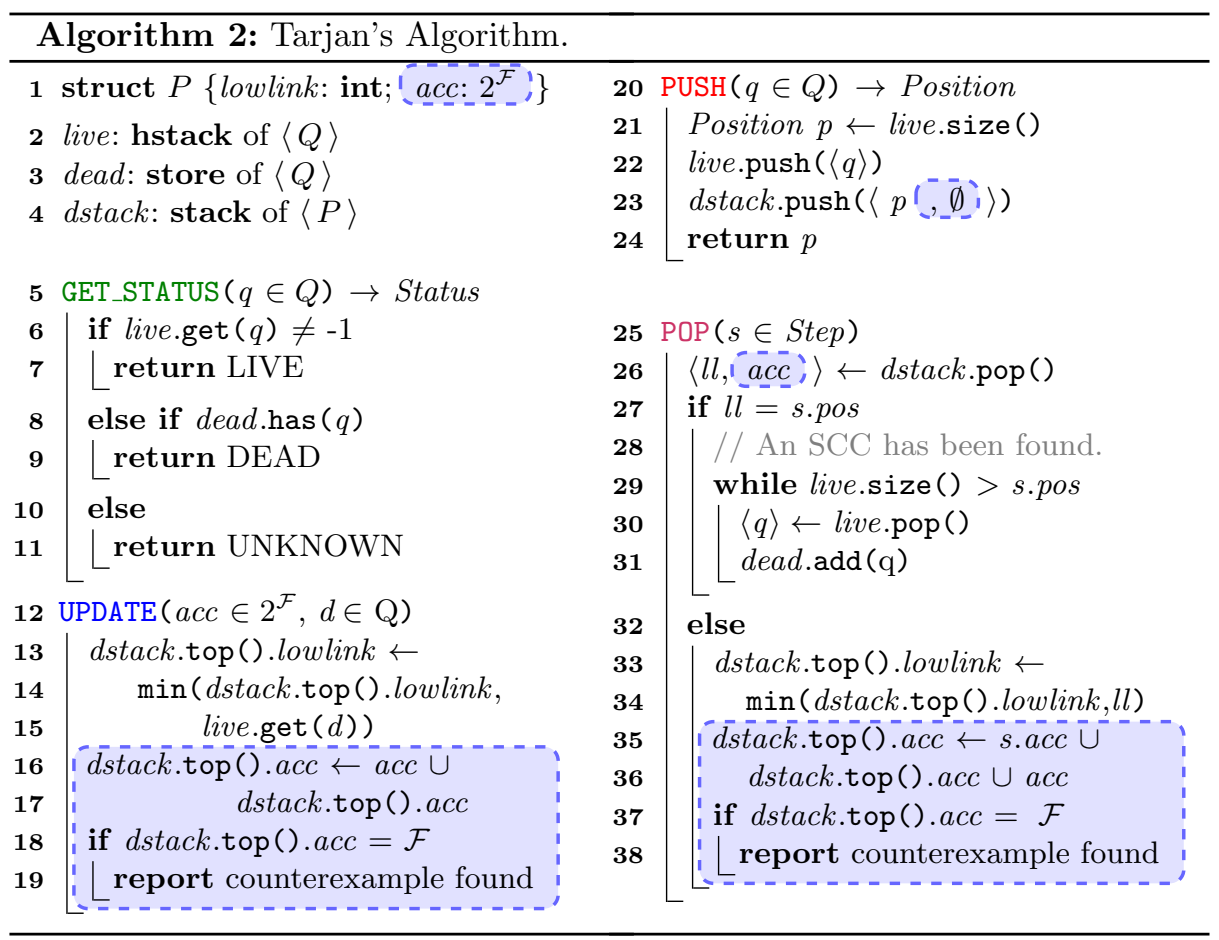

and the top of the stack. They can therefore be marked as DEAD by unwinding this stack, without exploring the graph.

Because a lowlink is only useful for states on $d f s$, it seems judicious to store it into a dedicated stack denoted dstack. This stack stores elements of the form $\langle$ lowlink, acc $\rangle$ where acc is only useful when doing an emptiness check.

As the states on $d f s$ are LIVE, they are simply identified by their position on live. We use this position instead of the DFS number when initializing lowlink.

To implement this live stack, we introduce a data structure hstack that stores all LIVE states and can be manipulated like a stack (with push and pop). To find the status of a state, we need to check whether it belongs to this hstack, therefore this structure is equipped with a get method that looks up a hash table to return the position associated to a given state, or -1 for missing states.

The set of DEAD states are represented by a separate data structure that support the following two operations: add and has with obvious semantics. As we shall discuss in Section 6, bit-state hashing and state-space caching can be implemented by redefining these operations.

Algorithm 2 presents our refactoring of the original Tarjan's algorithm to fit in the framework of Algorithm 1. The blue dashed boxes should be ignored on first read: they represent the parts to add to turn this SCC-enumeration algorithm into an emptiness check for TGBA. 
Because LIVE and DEAD states are respectively stored in live and dead, GET_STATUS can easily report all other states as UNKNOWN.

As explained previously, the lowlinks are updated everytime a transition is backtracked, i.e., at lines 12-15 when backtracking a back-edge, and at 32-34 when backtracking a forward-edge inside an SCC. When POP detects the root of an SCC (line 27), it simply unwind live to mark all the SCC's states as DEAD.

\section{$3.2 \quad$ Emptiness check}

Adding the blue dashed boxes will turn the SCC enumeration algorithm into an emptiness check algorithm. Each LIVE state on $d f s$ is now associated to an empty set of acceptance mark at line 1 . This set is updated each time an edge intern to an SCC is backtracked, at lines $16-17$ and $35-36$. These backtracking updates will ultimately propagate to the root, the set of all acceptance marks present in the SCC. Therefore, in the worse case, an accepting SCC will be detected when the root is popped, but it may happens earlier if one of the intermediate set is equal to $\mathcal{F}$ (hence the tests on lines 18 and 38).

To our knowledge, the only existing emptiness check based on Tarjan's algorithm has been proposed by Geldenhuys and Valmari [11]. Their algorithm targets only degeneralized Büchi automata (one acceptance mark), so they may have to explore a larger automaton that we do. However their algorithm works quite differently from this one: they maintain the lowlink for each LIVE state and a stack of LIVE accepting states (it would work for transition-based acceptance too) and they are therefore able to report a counterexample as soon as they close an accepting cycle, while our algorithm would have to wait for an accepting transition to be popped. This detection could be done earlier by associating an acceptance set to each element of live. As we target memory efficience this solution has not been retained.

\section{Dijkstra-based Algorithms}

\subsection{SCC Computation}

Intuitively, Dijkstra's algorithm [6] maintains a stack of SCCs of the subgraph that has been explored. Everytime a back-edge is found, closing a cycle, the SCCs forming that cycle are merged.

In practice, Algorithm 3 (without the green dotted boxes) actually manages three stacks: live, the set of LIVE states; $d f s$, the subset of live that are on the DFS search path, represented - as in the previous section - by a stack of positions in live; and roots, the stack of SCC roots, stored as positions in the $d f s$ stack. When given two consecutive roots, roots $[i]$ and roots $[i+1]$, the set of states belonging to the SCC rooted in roots $[i]$, are the states at positions $d f s[$ roots $[i]] . p o s, \ldots, d f s[\operatorname{roots}[i+1]]$.pos -1 in live. This representation makes several operations efficient. Merging consecutive SCCs can be done by simply removing elements from roots (lines 18 and 21). Also, it possible to decide whether 


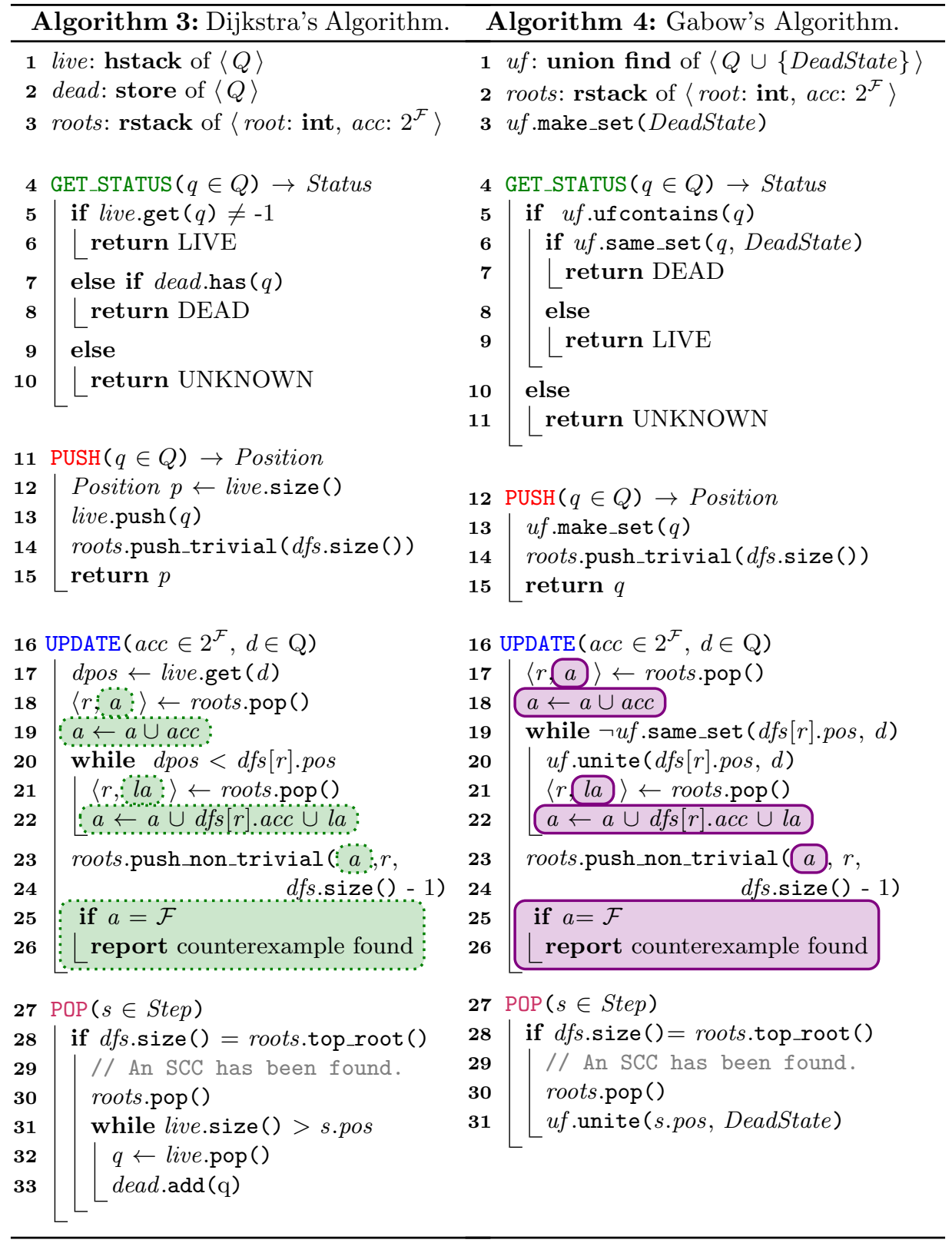

a state is a root of an SCC during POP: when the position pointed to by the top of the roots stack is equal to the size of $d f s$ (line 28) it means the state that has already been popped by the main DFS algorithm was a root. 


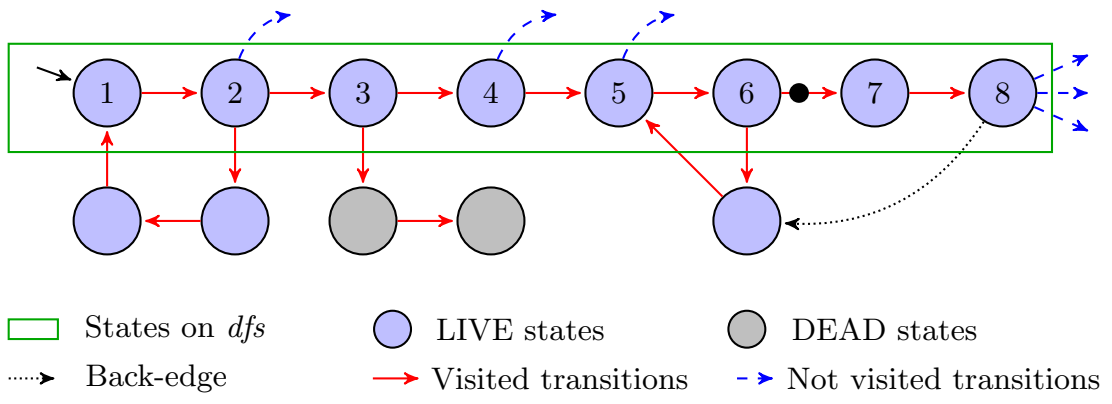

Before visiting back-edge

\begin{tabular}{|c|c|c|c|c|c|c|c|c|c|c|c|}
\hline \multirow[t]{2}{*}{ roots } & 1 & 3 & 4 & 5 & 7 & 8 & \multirow[t]{2}{*}{ position } & 1 & 3 & 4 & 5 \\
\hline & $\emptyset$ & $\emptyset$ & $\emptyset$ & $\emptyset$ & $\emptyset$ & $\emptyset$ & & $\emptyset$ & $\emptyset$ & $\emptyset$ & 0 \\
\hline \multirow{3}{*}{$\begin{array}{l}\text { roots } \\
\text { (compressed) }\end{array}$} & 2 & 4 & 6 & 8 & & & \multirow{3}{*}{$\begin{array}{c}\text { position } \\
\text { acc } \\
\text { trivial? }\end{array}$} & 2 & 4 & 8 & \\
\hline & $\phi$ & $\emptyset$ & $\bar{\emptyset}$ & $\emptyset$ & & & & $\emptyset$ & $\emptyset$ & 0 & \\
\hline & $\times$ & $\checkmark$ & $\times$ & $\checkmark$ & & & & $\times$ & $\checkmark$ & $x$ & \\
\hline
\end{tabular}

Fig. 1. Stack compression in action where numbers corresponds to DFS positions.

The roots stack is implemented with a structure called rstack that supports three operations: pop(), push_trivial(begin) and push_non_trivial(begin,end). The latter two distinguish whether the SCC being pushed is trivial or not. They can be implemented as a normal push(begin), but in Section 4.2 we will see how to use these to compress the stack. Initially, any newly visited state constitutes a trivial SCC (line 14) with respect to the explored part of the automaton; non-trivial SCCs are only created when merging SCCs because of a back-edge (line 24).

DEAD states are stored in a dead store as in the previous algorithm, and for the same reason.

\subsection{Compressing the roots Stack}

The roots stack represents two kinds of SCCs: trivial and non-trivial. We suggest to compress this stack by representing ranges of consecutive trivial SCCs in a single entry. Each stack entry should have an additional Boolean indicating whether it represents of range of trivial SCCs or a non-trivial SCC, and should store the position of the last state seen before moving to the next entry. Figure 1 shows the effect of this compression.

In the worst case, it appears that we are simply adding one extra bit per entry, but as we shall see in our experiments, merging consecutive trivial SCCs is really effective. 


\subsection{Emptiness checks}

Dijkstra's algorithm can be turned into a emptiness check by adding the green dotted boxes. Each SCC is associated to a set of acceptance marks that have been seen inside this SCC. When some SCCs are merged, their acceptance marks are merged along with the marks of the transitions between these SCCs (line 25-26). A counterexample can be reported as soon as this union is $\mathcal{F}$.

Several authors have devised emptiness-check algorithms using this principle $[1,5,12,14,10]$. In this scheme, the main DFS can also be adjusted to chose the next transition to visit among all the non-visited outgoing transitions of the topmost SCC $[1,5,14]$.

The algorithm proposed by Couvreur [4] is sometimes considered as a Dijkstrabased algorithm [12]: it replaces the live stack by a simple hash map (save a tiny bit of memory) and consequently has to rediscover the states that need to be marked DEAD during POP (loosing time). Nevertheless it fit perfectly into the generic canvas of Algorithm 1 and can easily be mixed with bitstate hashing and state space caching by using a dead store.

\section{Gabow-based Algorithms}

The POP operation of previous algorithms is costly because it has to visit all the states in top SCC to mark them as DEAD.

If we regard Dijkstra's algorithm as partitioning of the set of states, each (live) SCC corresponds to a class in this partition, and an additional class stores all DEAD states. Merging SCCs maps to unions of LIVE classes in this partition, while popping an SCCs should incur a union with the class of dead states.

This observation is the base of Gabow's suggestion [8] to use the Union-Find data structure [20] to discover the SCCs of a graph. In this data structure, a union operation can be achieved in near constant-time (or even constant-time for this particular application [9]), without enumerating all its states.

The Union-Find structure partitions the set $Q^{\prime}=Q \cup\{$ DeadState $\}$ where DeadState represent an extra artificial DEAD state, and offers the following methods: make_set $\left(s \in Q^{\prime}\right)$ creates a new class containing the state $s$; unite $\left(s_{1} \in\right.$ $\left.Q^{\prime}, s_{2} \in Q^{\prime}\right)$ makes the union between two classes given by their representatives $s_{1}$ and $s_{2}$; and same_set $\left(s_{1} \in Q^{\prime}, s_{2} \in Q^{\prime}\right)$ checks whether two states are in the same class.

Algorithm 4 follows the same schema as Algorithm 3, except that we have replaced live and dead, by the Union-Find structure uf, and that Positions stored in $d f s$ are now pointers to states. When the root of an SCC is popped (line 28), its class is merged with that of the artificial DeadState (line 31). GET_STATUS has to be updated to check deadness using this DeadState as well. UPDATE is done easily by uniting all classes representing the SCCs on the cycle.

The main difference with Dijkstra's algorithm is therefore that the use of unite in function POP dispenses from enumerating all states in the SCC. This approach remains compatible with the compression of the roots stack presented 
in Sec. 4.2, and can be turned into an emptiness check in the same way as Dijkstra (adding purple boxes).

As-is, this algorithm is neither compatible with bit-state hashing nor statespace caching, because there is no dead store. Compatibility with these techniques is possible, but tricky. We discuss it in the next Section.

\section{Bit-State Hashing \& State-Space Caching Compatibility}

Bit-state hashing [15] and state-space caching [13] are two techniques to save memory. In bit-state hashing, collisions in the hash table storing dead states are ignored, turning the algorithm into a semi-decision procedure. In state-space caching, dead state can be removed from the store at any moment, causing the algorithm to possibly revisit a state several times.

On Tarjan-based and Dijkstra-based algorithms, these techniques can be implemented by replacing the has and add methods of the dead store, implemented as a hash table. Note that for bit-state hashing, it is important to check the membership to live before the membership to dead in GET_STATUS.

When compatibility with these techniques is not required, we can forgot the use of this extra hash table, and actually store LIVE and DEAD states in the same table, using a extra bit to distinguish LIVE from DEAD. This saves a table lookup in GET_STATUS.

For Gabow's algorithm, compatibility with bit-state hashing and state-space caching is more tricky to achieve and we only give the intuition. First, the UnionFind data structure, which stores states in a vector, has to be made aware of what a DEAD state is: let us assume that the unite of line 31 is changed to make_dead. The first time make_dead is called, the states to be marked as DEAD are all at the end of the vector. The trick is to remember the frontier between LIVE and DEAD states in that vector. Then, every time a new singleton class is created with the make_set operation, we can reuse the slot of the first DEAD state (right after the frontier), and move that DEAD state to the DEAD store. GET_STATUS has to be updated as well.

Note that in this approach, the set of DEAD states is distributed in two structures: the end of the Union-Find vector, and the DEAD store, but only this store can be subject to bit-state hashing or state-space caching. However this approach still avoids the enumeration of states to mark them DEAD.

\section{Implementation Issues and Benchmarks}

All these approaches have been implemented in Spot [7]. The Union-Find structure of Gabow's algorithm uses common optimizations: "Immediate Parent Compression", "Link by Rank", "Path Compression", and "Memory Smart" [17].

When dead does not use bit-state hashing nor state-space caching techniques, an optimization consists in marking states as DEAD inside the live structure 


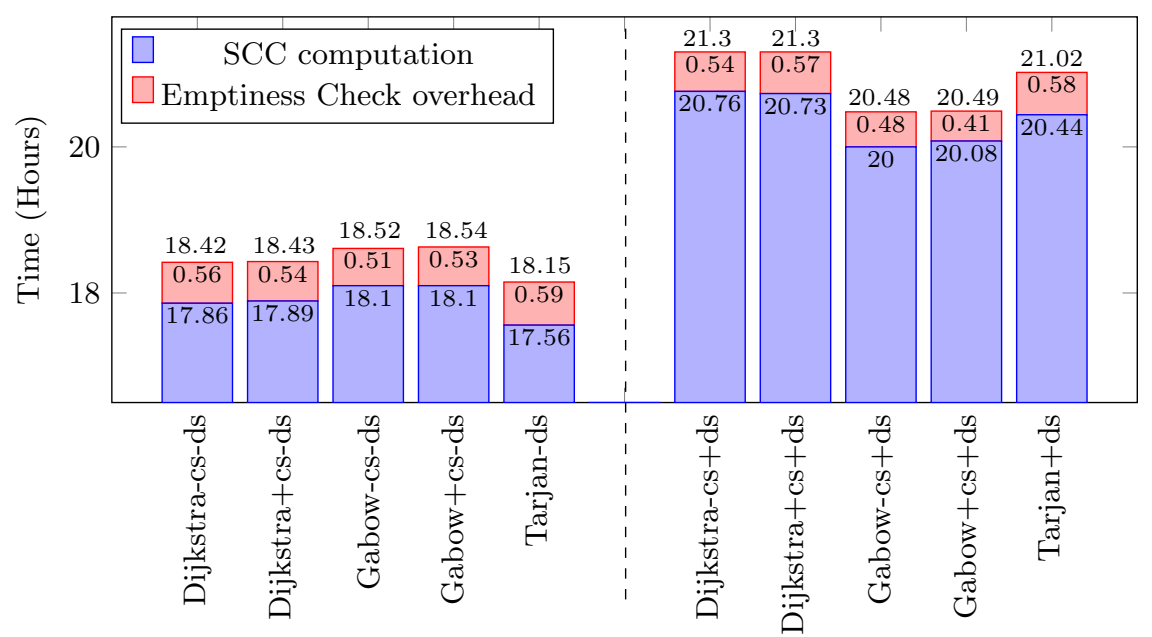

Fig. 2. Overhead of the emptiness checks over the SCC computations on 448 empty products. A total of $2.5 \times 10^{9}$ states, $17.3 \times 10^{9}$ transitions, and $10^{9}$ SCCs were visited.

rather than transferring it into dead during a POP. This optimization only requires a special value to tag a state DEAD. Its use is denoted by -ds in tables, while the use of a dedicated dead store (as presented previously) is denoted by + ds. Similarly, + cs and $-c s$ indicate whether the roots stack optimization (Sec. 4.2) is enabled or disabled.

The models we use come from the BEEM benchmark [18]. We generate the corresponding system automata using a version of DiVinE 2.4 patched by the LTSmin team. ${ }^{5}$ Because there are too few LTL formulas supplied by the BEEM benchmark, we opted to generate random formulas for each model. We computed a total number of 860 formulas. ${ }^{6}$

A formula and a model generate a product that may be either empty (the formula is verified) or non-empty (a counterexample exists). To decide that a product is empty, any emptiness check has to explore all the reachable states of the product. Conversely, a non-empty product can be reported as soon as an accepting SCC is detected, avoiding the need to explore the entire product. In our implementation, all algorithms use the same generic DFS traversal and thus visit transitions in the same order.

Among our formulas, 412 result in non-empty product with the model. The remaining 448 formulas, associated to empty products, were selected so that the emptiness check algorithms would take at least 10 seconds on an Intel(R) 64-bit $\mathrm{Xeon}(\mathrm{R}) @ 2.00 \mathrm{GHz}$ with 64GB of RAM.

\footnotetext{
${ }^{5}$ http://fmt.cs.utwente.nl/tools/ltsmin/\#divine

${ }^{6}$ For a detailed description of our setup, including selected models and formulas, see http://move.lip6.fr/ Etienne.Renault/benchs/LPAR-2013/results_scc.html.
} 
Figure 2 shows the execution time of all the emptiness check variants presented in this paper (with or without dead store, with or without compressed roots stack). To measure the overhead of the emptiness check over the SCC computation, we only focus on empty products.

For each bar the lower part represents the SCC computation time while the upper part corresponds to the overhead induced by the emptiness check. The total execution time is indicated atop the bar. The 5 rightmost bars show the emptiness check with a dead store enabled $(+\mathrm{ds})$ while the 5 leftmost bars have it disabled (-ds).

For the same + ds/-ds setting, all execution times are very close, and the emptiness check overhead is $3 \%$ on the average.

When the dead store is disabled, Tarjan is slightly better than Dijkstra, which is itself slightly better than Gabow. Activating the dead store generate an overhead of about $15 \%$, and is more favorable to Gabow. This latter point is due to the fact that our handling of the dead store for Gabow's algorithm, described in Section 6, will transfer less states from live to dead; this reduces the overhead to $10 \%$ only.

Table 1 reports the memory consumption, based on the size of the data structures used. As for time measurement, these experiments only focuses on verified formulas. The second column gives the formula that computes memory consumption at any time. The third column shows the peak we observed while running our experiments.

From that figure it appears that Dijkstra is the most memory efficient algorithm. Indeed the stack used by Dijkstra is a subset of the $d f s$ stack while the dstack of Tarjan, storing a lowlink and an acceptance set for each element, follows the variations of $d f s$. Gabow's algorithm requires more memory than the two others since it has to maintain the whole structure of the Union-Find. The use of a dead store significantly reduces memory consumption (up to 17\%).

When bit-state hashing or state-space caching are used, the size of $\mid$ dead $\mid$ can be fixed arbitrarily, allowing an even greater reduction.

Table 2 reports the the cumulated number of transitions, states and SCC visited by each algorithm for the 412-non empty products. We use this table to compare how quickly each algorithm reports a counterexample.

Gabow's and Dijkstra's algorithms have identical results since they both report a counterexample when a cycle is closed during UPDATE, while Tarjan's algorithm may delay the report of a counterexample to a later POP and visit several states until then. Nonetheless this difference is very small in our experiment: less than $1 \%$ additional states, transitions or SCCs have been visited. This negligible difference justifies our decision not to store an additional acceptance set in each element of live to report counterexamples earlier in Tarjan's algorithm, as discussed at the end of Sec. 3.2.

Table 3 presents the impact of the lazy transfer into dead proposed for Gabow's algorithm. We observe that only half the states are transferred to dead; this means that the remaining states have been preserved in the DEAD part of the Union-Find structure. This explains the gain observed from Fig. 2. 
Table 1. Comparison of memory consumption for emptiness check algorithms on the 448 empty products. $\mid$ roots $\mid$ (resp. $\mid$ uf $|$,$| dstack |$,$| dead \mid)$ denotes the number of elements in rstack (resp. uf, dstack, dead). As rstack elements are pairs (root, acc), we count the memory consumption as $2 \mid$ roots $\mid$ words. The additional bit required for each element of the compressed stack is not accounted for. Since live is constructed using an hashmap and a stack, we distinguish these sizes with $\mid$ live $_{\text {stack }} \mid$ and $\mid$ live $_{\text {hash }} \mid$ : they differ when no dead store is used.

\begin{tabular}{|c|c|c|}
\hline Algorithm & Memory consumption (words) & Observed peak \\
\hline Dijkstra-cs-ds & \multirow{2}{*}{$2 \mid$ roots $|+|$ live $_{\text {stack }}|+2|$ live $_{\text {hash }} \mid$} & 6225414223 \\
\hline Dijkstra+cs-ds & & 6225411039 \\
\hline Gabow-cs-ds & \multirow{2}{*}{$2|\operatorname{roots}|+3|u f|$} & 7364856119 \\
\hline Gabow+cs-ds & & 7364854033 \\
\hline Tarjan-ds & $2 \mid$ dstack $|+|$ live $_{\text {stack }}|+2|$ live $_{\text {hash }} \mid$ & 6325991684 \\
\hline Dijkstra-cs+ds & \multirow{2}{*}{$2 \mid$ roots $|+|$ live $_{\text {stack }}|+2|$ live $_{\text {hash }}|+|$ dead $\mid$} & 5160440344 \\
\hline Dijkstra $+\mathrm{cs}+\mathrm{ds}$ & & 5160435523 \\
\hline Gabow-cs+ds & \multirow{2}{*}{$2 \mid$ roots $|+4| u f|+|$ dead $\mid$} & 6608486024 \\
\hline Gabow $+\mathrm{cs}+\mathrm{ds}$ & & 6608482885 \\
\hline Tarjan+ds & $2 \mid$ dstack $|+|$ live $_{\text {stack }}|+2|$ live $_{\text {hash }}|+|$ dead $\mid$ & 5265484149 \\
\hline
\end{tabular}

Table 2. Cumulated States, transitions, and SCCs visited by each emptiness check on the 412 non-empty products.

\begin{tabular}{|c|c|c|c|}
\hline & Transitions & States & SCCs \\
\hline Tarjan & 534471068 & 67230381 & 34622772 \\
\hline Dijkstra/Gabow & 534338119 & 67187854 & 34582459 \\
\hline
\end{tabular}

Table 3. Impact of the dead strategy of Gabow's algorithm on the 448 empty products.

\begin{tabular}{lccc} 
& $\begin{array}{c}\text { Max. } \\
\text { dead peak }\end{array}$ & $\begin{array}{c}\text { Cumulated } \\
\text { dead peak }\end{array}$ \\
\cline { 2 - 2 } Tarjan/Dijkstra (+ds) & 29098013 & & 2454950318 \\
Gabow (+ds) & 21430297 & & 1070440670
\end{tabular}

Table 4. Impact of the compressed roots stack on the 448 empty products.

\begin{tabular}{lccc} 
& $\begin{array}{c}\text { Max. } \\
\text { roots } \text { peak }\end{array}$ & & $\begin{array}{c}\text { Cumulated } \\
\text { roots peak }\end{array}$ \\
\cline { 2 - 2 } Dijkstra/Gabow (-cs) & 456 & & 98322 \\
Dijkstra/Gabow (+cs) & 119 & & 8188
\end{tabular}

This observation also suggests that a similar optimization could be applied to Tarjan's and Dijkstra's algorithms: each time the live stack is reduced, the residual space (the free list) can be reused to store DEAD states temporarily. 
Table 4 shows the impacts of the compression technique proposed in Sec. 4.2. It allows a tenfold memory reduction without run-time overhead according to Fig. 2. Note that such a compression technique is independent of the emptiness check layer, but may apply to Dijkstra's and Gabow's SCC computations.

In Sec. 5, we suggested that using Union-Find was an efficient way to mark all states of an SCC as DEAD in a single operation. Unfortunately, Fig. 2 reveals that these gains are offset by the inherent cost of maintaining the UnionFind structure. Our implementation of the Union-Find uses classical optimizations [17] but we have yet to investiguate wether performances could be improved by the use of a data structure dedicated to the case where each union only concern the last SCCs [9].

\section{Conclusion}

This paper proposed an overview of existing SCC enumeration algorithms and proposed a generic canvas to transform them into emptiness checks for TGBA.

This lead us to define two new emptiness checks. One is based on Tarjan; it differs from [11] in that it is more memory efficient and generalized. Another one is based on Gabow's suggestion to use the Union-Find data structure: our results with that data structure are mixed, but as far as we know, this is the first time this data structure is used for emptiness check.

We also introduced a couple of optimizations. For Dijkstra's and Gabow's emptiness checks we suggest to compress the roots stack to save some memory. Additionally, we discussed a strategy to transfer DEAD state from the UnionFind structure to the dead store lazily, resulting in an important gain of time, and this strategy could also be applied to the other algorithms.

We have several leads for future work. One would be to devise a compression technique for the stack of lowlink (dstack) used by Tarjan's algorithm to make it more competitive to Dijkstra's algorithm (currently more memory-efficient). Furthermore, the compaction of the live stack suggested by Nuutila and SoisalonSoininen [16] for Tarjan's algorithm could be adapted to Dijkstra's algorithm and (with a more work) to Gabow's. Another idea would be to study the various ways to extract counterexamples from these algorithms; the procedure suggested by Couvreur et al. [5] would work for Dijkstra and Gabow but should not be difficult to adapt to Tarjan. Finally, we would like to investigate the possibility to parallelize these emptiness checks. There are very few parallel emptiness checks based on SCC computations [2], however as Tarjan and Dijkstra use different data structure than Gabow, may be one of them will be more favorable to a parallel setup.

\section{References}

[1] R. Alur, S. Chaudhuri, K. Etessami, and P. Madhusudan. On-the-fly reachability and cycle detection for recursive state machines. In TACAS'05, vol. 3440 of LNCS, pp. 61-76. Springer, April 2005. 
[2] I. Černá and R. Pelánek. Distributed explicit fair cycle detection (set based approach). In SPIN'03, vol. 2648 of LNCS, pp. 49-73. Springer, May 2003.

[3] C. Courcoubetis, M. Y. Vardi, P. Wolper, and M. Yannakakis. Memory-efficient algorithm for the verification of temporal properties. In CAV'90, vol. 531 of LNCS, pp. 233-242. Springer, 1991.

[4] J.-M. Couvreur. On-the-fly verification of temporal logic. In FM'99, vol. 1708 of LNCS, pp. 253-271, Sept. 1999. Springer.

[5] J.-M. Couvreur, A. Duret-Lutz, and D. Poitrenaud. On-the-fly emptiness checks for generalized Büchi automata. In SPIN'05, vol. 3639 of LNCS, pp. 143-158. Springer, Aug. 2005.

[6] E. W. Dijkstra. EWD 376: Finding the maximum strong components in a directed graph. http://www.cs.utexas.edu/users/EWD/ewd03xx/EWD376. PDF, May 1973.

[7] A. Duret-Lutz and D. Poitrenaud. SPOT: an Extensible Model Checking Library using Transition-based Generalized Büchi Automata. In MASCOTS'04, pp. 76-83, Oct. 2004. IEEE Computer Society Press.

[8] H. N. Gabow. Path-based depth-first search for strong and biconnected components. Information Processing Letters, 74(3-4):107-114, February 2000.

[9] H. N. Gabow and R. E. Tarjan. A linear-time algorithm for a special case of disjoint set union. In STOC'83, pp. 246-251, 1983. ACM.

[10] A. Gaiser and S. Schwoon. Comparison of algorithms for checking emptiness on Büchi automata. In MEMICS'09, vol. 13 of OASICS. Schloss Dagstuhl, LeibnizZentrum fuer Informatik, Germany, Nov. 2009.

[11] J. Geldenhuys and A. Valmari. Tarjan's algorithm makes on-the-fly LTL verification more efficient. In TACAS'04, vol. 2988 of LNCS, pp. 205-219. Springer, 2004 .

[12] J. Geldenhuys and A. Valmari. More efficient on-the-fly LTL verification with Tarjan's algorithm. Theoretical Computer Science, 345(1):60-82, Nov. 2005.

[13] P. Godefroid, G. J. Holzmann, and D. Pirottin. State space caching revisited. In CAV'92, vol. 663 of LNCS, pp. 178-191. Springer, 1992.

[14] H. Hansen and J. Geldenhuys. Cheap and small counterexamples. In SEFM'08, pp. 53-62. IEEE Computer Society, Nov. 2008.

[15] G. J. Holzmann. On limits and possibilities of automated protocol analysis. In PSTV'87, pp. 339-344. North-Holland, May 1987.

[16] E. Nuutila and E. Soisalon-Soininen. On finding the strongly connected components in a directed graph. Information Processing Letters, 49(1):9-14, Jan. 1994.

[17] M. M. A. Patwary, J. R. S. Blair, and F. Manne. Experiments on union-find algorithms for the disjoint-set data structure. In SEA'10, vol. 6049 of LNCS, pp. 411-423. Springer, 2010.

[18] R. Pelánek. BEEM: benchmarks for explicit model checkers. In Proc. of the 14th international SPIN conference on Model checking software, Lecture Notes in Computer Science, pp. 263-267. Springer, 2007.

[19] R. Tarjan. Depth-first search and linear graph algorithms. SIAM Journal on Computing, 1(2):146-160, 1972.

[20] R. E. Tarjan. Efficiency of a good but not linear set union algorithm. Journal of the ACM (JACM), 22(2):215-225, Apr. 1975. 